\title{
Tumor-infiltration of T-lymphocytes is inversely correlated with clinicopathologic factors in endometrial adenocarcinoma
}

\author{
In Kook Jung ${ }^{1 *}$, Sun Sook Kim², Dong Soo Suh', Ki Hyung Kim', Chang Hoon Lee ${ }^{3}$, Man Soo Yoon ${ }^{1}$ \\ ${ }^{1}$ Department of Obstetrics and Gynecology, Pusan National University Hospital, Pusan National University School of Medicine, and Biomedical \\ Research Institute and Pusan Cancer Center, ${ }^{2}$ Department of Medicine, Pusan National University Graduate School of Medicine, ${ }^{3}$ Department of \\ Pathology, Pusan National University School of Medicine, Busan, Korea
}

\section{Objective}

The aim of this study was to determine the distribution of T-lymphocytes and their relationship with clinicopathologic factors in endometrial carcinoma.

\section{Methods}

Samples were collected from 89 patients with endometrial endometrioid adenocarcinoma treated in Pusan National University Hospital from 2004 to 2011. Normal endometrial tissues were obtained from 30 hysterectomized women with benign adnexal masses and served as controls. Paraffin-embedded sections were immunohistochemically stained for CD8 (cytotoxic) and CD4 (helper) T-lymphocytes. The relationship of these cells with stage, histological grade, myometrial invasion, and lymph node metastasis was analyzed.

\section{Results}

The proportion of CD8+ and CD4+ lymphocytes in the endometrial endometrioid adenocarcinoma tissues was $67.4 \%$ $(60 / 89)$ and $44.9 \%(40 / 89)$, respectively, which was significantly higher $(P<0.05)$ than in the control group. The extent of CD8+ lymphocyte expression was negatively correlated with histologic grade, myometrial invasion, and lymph node metastasis. The proportion of infiltration of the CD4+ lymphocytes was negatively correlated with histologic grade and myometrial invasion.

\section{Conclusion}

The high rate of infiltration of T-lymphocytes was negatively correlated with histologic grade, myometrial invasion, and lymph node metastasis. Our findings suggest that tumor-infiltrating T-lymphocytes may be used as pathologic prognostic factors in endometrial carcinoma.

Keywords: Clinicopathologic factors; Endometrial carcinoma; T-lymphocytes

\section{Introduction}

Endometrial adenocarcinoma is the most common gynecologic malignancy in the United States and Western Europe. In Korea, the incidence of endometrial cancer has been increasing in recent years: the age standardized incidence rate per 100,000 during 2010 was 5.0 [1]. The recent increasing prevalence of risk factors is expected to result in a further increase in the incidence of this cancer.

Patients with endometrial cancer have a good prognosis due to early detection resulting from postmenopausal
Received: 2013.8.16. Revised: 2014.1.1. Accepted: 2014.1.18.

Corresponding author: Ki Hyung Kim

Department of Obstetrics and Gynecology, Pusan National University School of Medicine, 179 Gudeok-ro, Seo-gu, Busan 602-739, Korea

Tel: +82-51-240-7287 Fax: +82-51-248-2384

E-mail: ghkim@pusan.ac.kr

*These two authors contributed equally to this work.

Articles published in Obstet Gynecol Sci are open-access, distributed under the terms of the Creative Commons Attribution Non-Commercial License (http://creativecommons. org/licenses/by-nc/3.0/) which permits unrestricted non-commercial use, distribution, and reproduction in any medium, provided the original work is properly cited.

Copyright $\odot 2014$ Korean Society of Obstetrics and Gynecology 


\section{Obstetrics \& Gynecology Science}

In Kook Jung, et al. T lymphocytes as prognostic factors

bleeding. However, the prognosis for recurrent or metastatic endometrial cancer remains poor. An essential step for the improvement of clinical outcomes is to understand the processes that inhibit and stimulate cancer progression [2].

Known prognostic factors in endometrial carcinoma include tumor stage, histologic grade, subtype, and vascular invasion [3]. In addition, changes in the immune system in the tumor microenvironment are considered one of the important factors in the development and progression of cancer. Tumorinfiltrating lymphocytes are often found in human cancer. Increasing evidence indicates that the high rate of infiltration of T-lymphocytes has been reported to be an indicator of good prognosis. A positive association between infiltrating CD8+ T cells and prognosis has been reported in various solid cancers such as colorectal and ovarian cancer $[4,5]$. However, the presence of tumor infiltrating lymphocytes (TILs) has not been fully assessed in endometrial cancer.

In the present study, we analyzed the tumor infiltration of CD8+ and CD4+ lymphocytes to determine whether the presence of T-lymphocytes was associated with prognostic factors in endometrial carcinoma.

\section{Materials and methods}

\section{Patients and tissue samples}

A total of 89 patients diagnosed with endometrial endometrioid carcinoma who underwent surgery from 2004 to 2011 were selected from the archives of the Pusan National University Hospital for this study. All patients underwent a total abdominal hysterectomy and a bilateral salpingooophorectomy (BSO). Pelvic and/or para-aortic lymph node dissection was performed in the patients with more advanced disease or unfavorable histologic grade. Hematoxylin and eosin (H\&E) stained sections were reviewed and reclassified by World Health Organization (WHO) guidelines. Histologic type, grade of differentiation, stage, and depth of myometrial invasion had previously been evaluated in the tissue materials. This study was approved by the Ethical Review Committee of Pusan National University Hospital.

\section{Immunohistochemistry}

The tissue specimens were fixed in 10\% formalin and embedded in paraffin. Sections, $4 \mu \mathrm{m}$ in thickness, were deparaffinized in xylene and rehydrated through a series of graded ethanol. Endogenous peroxidase activity was blocked by incubation with 3\% hydrogen peroxide in methanol for 10 minutes. Antigen retrieval was performed by microwaving the slides in citrate buffer ( $\mathrm{pH}$ 6.0). The sections were then incubated at $4^{\circ} \mathrm{C}$ overnight with anti-Human CD4 antibody (rabbit monoclonal, SP35, 1:50; Spring Bioscience, Pleasanton, CA, USA), anti-Human CD8 antibody (mouse monoclonal, C8/144B, Dako, Glostrup, Denmark). Immunoreactivity was visualized using 3,3'-diaminobenzidine (DAB, Dako-Cytomation, Glostrup). Slides were counterstained with Meyer's hematoxylin. Human tonsil tissue for CD4 and skin for CD8 were used as positive controls.

\section{Evaluation of staining}

Each slide was evaluated independently by two pathologists who were blinded to clinical and outcome data. Three independent areas with the most abundant lymphocyte infiltration were selected, and the stromal lymphocytes were independently counted in each microscopic field at $\times 200$. The average count for three areas was accepted as the number of TILs in each case [6].

\section{Statistical analysis}

Statistical analyses were conducted using SPSS ver. 15.0 (SPSS Inc., Chicago, IL, USA). The $\chi^{2}$ test and Fisher exact test were used to evaluate the correlation between the expression of CD4 and CD8 and the clinicopathologic parameters. $P$-values of $<0.05$ were considered statistically significant.

\section{Results}

\section{Patients' characteristics}

The clinicopathological characteristics of the 89 patients are presented in Table 1. The mean age of the patients was 61 years (range, 40-78 years). Patients with endometrial cancer included 12 with premenopausal status and 77 with postmenopausal status. Clinical follow-up was available for all cases. The median follow-up period was 64 months (range, 8-84 months). Overall, $86.5 \%$ of the patients had early-stage disease $(\mathrm{I}, \mathrm{II})$ and $13.5 \%$ had advanced-stage disease. In addition, $40.4 \%$ had well-differentiated cancer (G1), 32.65\% had moderately differentiated cancer (G2), and $26.9 \%$ had poorly differentiated cancer (G3). None of the patients had undergone radiation or chemotherapy before surgery. Treatment 


\title{
Obstetrics \& Gynecology Science
}

\author{
Vol. 57, No. 4, 2014
}

Table 1. Clinicopathological characteristics of the patients with endometrial carcinoma

\begin{tabular}{|lc|}
\hline Variable & No. of patients (\%) \\
\hline Age $(\mathrm{yr})$ & $57(64.0)$ \\
$>60$ & $32(36.0)$ \\
Menopause & \\
Pre- & $12(13.5)$ \\
Post- & $77(86.5)$ \\
Body mass index $\left(\mathrm{kg} / \mathrm{cm}^{2}\right)$ & \\
$\leq 25$ & $27(30.3)$ \\
$>25$ & $62(69.7)$ \\
FIGO grade & \\
G1 & $36(40.0)$ \\
G2 & $29(32.6)$ \\
G3 & $24(27.4)$ \\
FIGO stage & \\
I & $64(71.9)$ \\
II & $13(14.6)$ \\
III & $10(11.2)$ \\
IV & $2(2.3)$ \\
\hline
\end{tabular}

FIGO, International Federation of Gynecology and Obstetrics. consisted of total hysterectomy and a BSO alone (30.3\%), or total hysterectomy and BSO and pelvic and/or para-aortic lymph node dissection (69.7\%). No patients had remaining macroscopic tumors at the time of surgery.

\section{CD8, CD4 infiltration into endometrial carcinoma tissues}

CD8 and CD4 antigens were stained in endometrial carcinoma tissues. The distribution pattern of CD8+, CD4+ lymphocytes was similar to that of lymphocytes as determined by conventional H\&E staining. Lymphocytic infiltration of CD8+, CD4+ was observed more frequently in the stroma surrounding tumors than within the tumors. The patients were divided into 2 groups based on the median CD8+ or CD4+ cell counts. The positive/high cell count group consisted of patients with $\geq 40$ stromal CD8+ or CD4+ T-lymphocytes in a microscopic field at $\times 200[6,7]$. The number of stromal CD8+ cells in positive group (range, 42-70; median, 50.3) was significantly higher $(P<0.01)$ than in negative group (range, 14-32; median, 26.3) and the number of stromal CD4+ cells in positive group (range, 44-82; median, 53.0) was also significantly higher $(P<0.01)$ than in negative group (range, 15-34; median, 24.7). The proportions of CD8+ and CD4+ lymphocytes in the endometrial adenocarcinoma were $67.4 \%$

Table 2. Association of tumor-infiltrating lymphocytes with clinicopathological parameters

\begin{tabular}{|c|c|c|c|c|c|c|}
\hline \multirow{2}{*}{ Variable } & \multicolumn{3}{|c|}{ CD4+ lymphocytes } & \multicolumn{3}{|c|}{ CD8+ lymphocytes } \\
\hline & High & Low & $P$-value ${ }^{\text {a) }}$ & High & Low & $P$-value ${ }^{\text {a) }}$ \\
\hline LN metastasis ${ }^{b)}$ & & & 0.136 & & & $0.002^{c}$ \\
\hline Negative & $30(42.3)$ & $41(57.7)$ & & $59(72.8)$ & $22(27.2)$ & \\
\hline Positive & $10(55.6)$ & $8(44.4)$ & & $1(12.5)$ & $7(87.5)$ & \\
\hline Myometrial invasion & & & 0.001 & & & $<0.001$ \\
\hline$<1 / 2$ & $35(57.4)$ & $26(42.6)$ & & $50(81.9)$ & $11(18.1)$ & \\
\hline$>1 / 2$ & $5(17.9)$ & $23(82.1)$ & & $10(35.7)$ & $18(64.3)$ & \\
\hline FIGO grade & & & 0.041 & & & $<0.001$ \\
\hline G1 & $22(61.1)$ & $14(38.9)$ & & $32(88.8)$ & $4(11.2)$ & \\
\hline G2 & $10(34.5)$ & $19(65.5)$ & & $18(62.1)$ & $11(37.9)$ & \\
\hline G3 & $8(33.3)$ & $16(66.7$ & & $10(41.6)$ & $14(58.4)$ & \\
\hline FIGO stage & & & 0.311 & & & $0.052^{c}$ \\
\hline$|-| \mid$ & $36(46.8)$ & $41(53.2)$ & & $55(71.4)$ & $22(28.6)$ & \\
\hline III-IV & $4(33.3)$ & $8(66.7)$ & & $5(41.6)$ & $7(58.4)$ & \\
\hline
\end{tabular}

Values are presented as number (\%).

LN, lymph node; FIGO, International Federation of Gynecology and Obstetrics.

${ }^{\text {a) }} P$-values were calculated using the chi square-test; Bold signifies $P<0.05 .{ }^{\text {b) }}$ Analyzed the positivities of samples in the patients with surgically proven LN pathology; ${ }^{\text {c) }}$ isher exact test. 


\section{Obstetrics \& Gynecology Science}

In Kook Jung, et al. T lymphocytes as prognostic factors

CD4

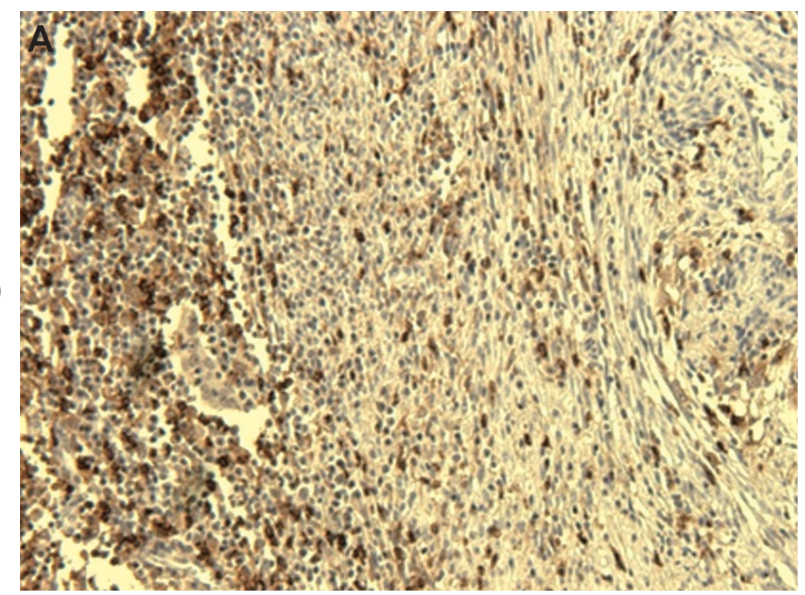

$(-)$

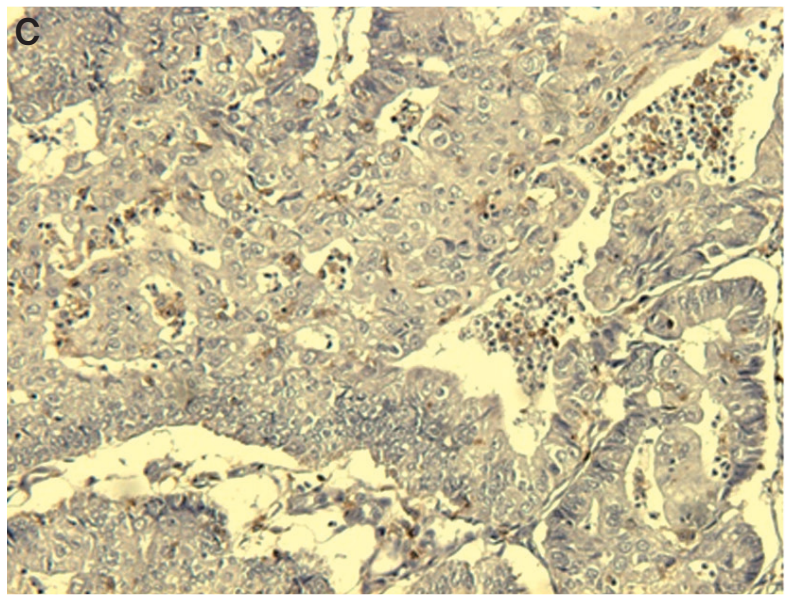

$(+)$

Control

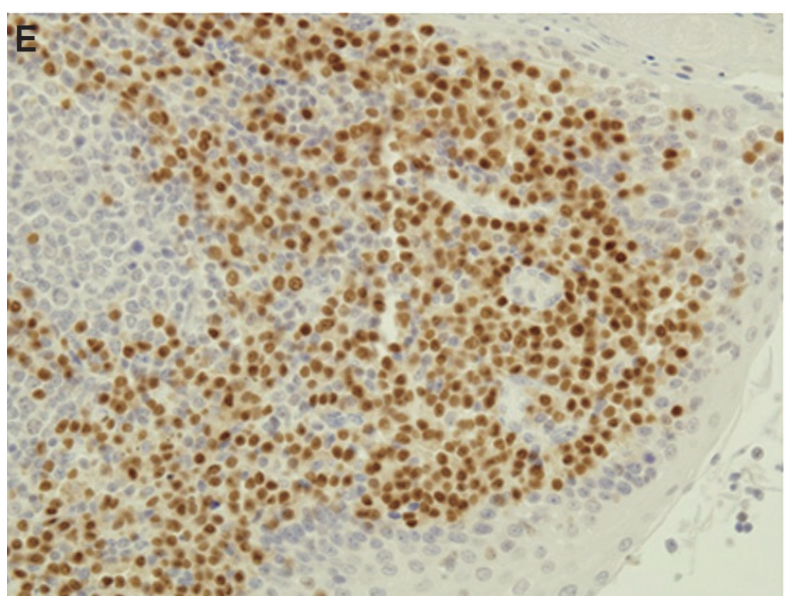

CD8
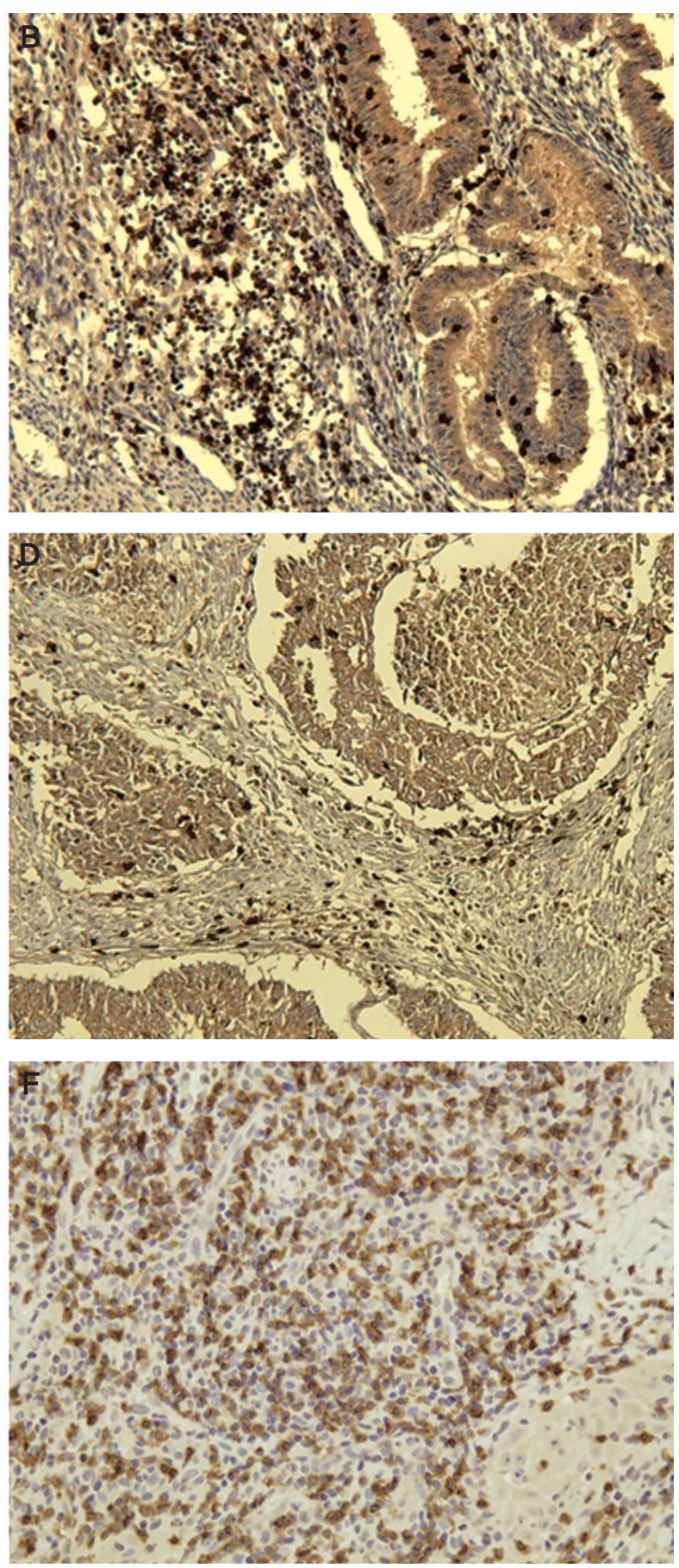

Fig. 1. Expression and histological distribution of $C D 4+$ and $C D 8+T$-lymphocytes in primary endometrial carcinoma specimens. Representative patient tissues were highly stained for CD4 (A) and CD8 (B). (C) and (D) were patient tissues with low CD4 or CD8 cell count. Human tonsil for CD4 (E) and skin for CD8 (F) were used as positive controls.

(60/89) and $44.9 \%(40 / 89)$, respectively, which were significantly higher $(P<0.001)$ than in the control group.
3. CD8, CD4 infiltration and clinicopathological factors

Fig. 1 shows a representative case of endometrioid endometrial carcinoma that illustrates the distribution pattern of 


\section{Obstetrics \& Gynecology Science}

Vol. 57, No. 4, 2014
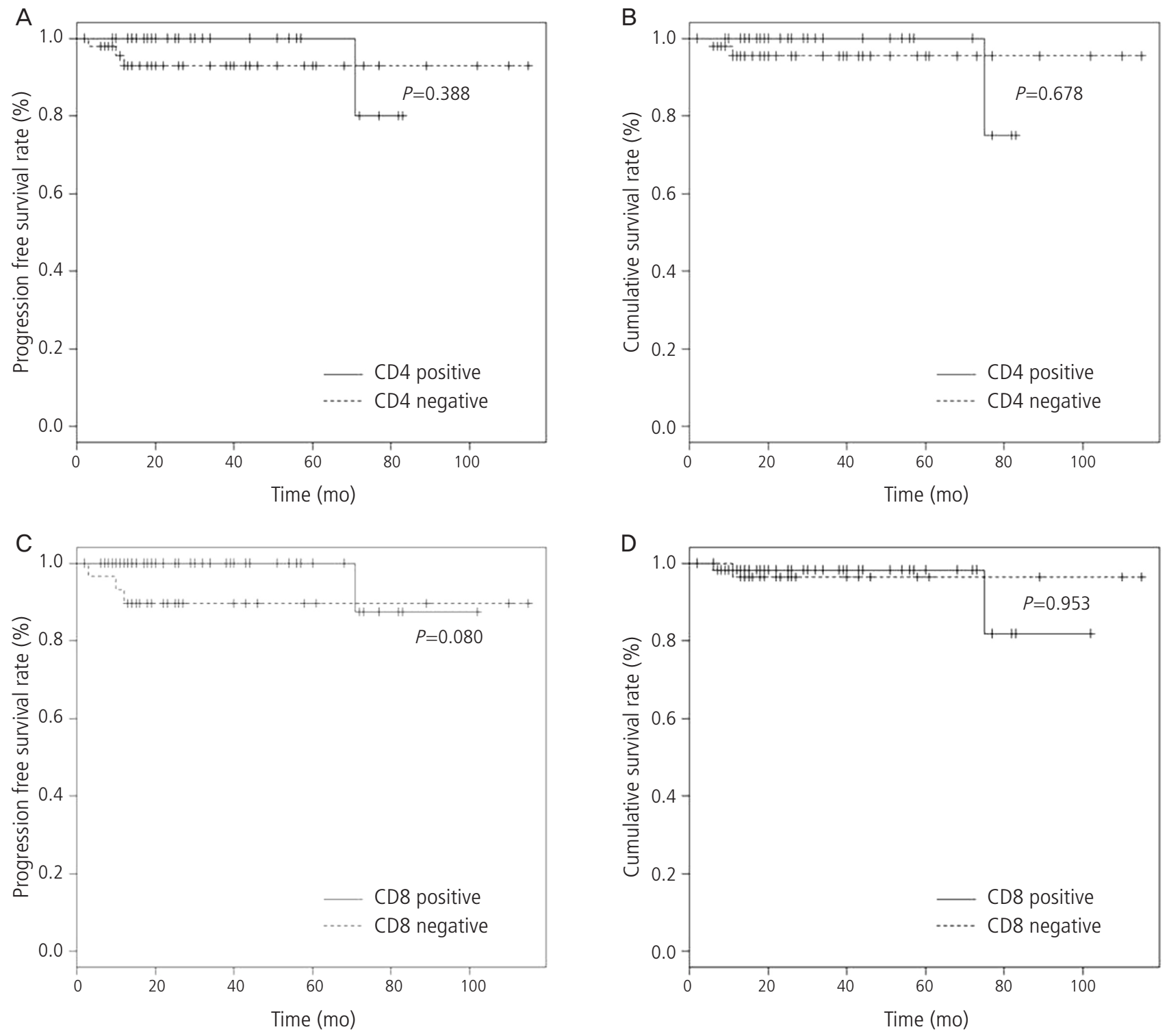

Fig. 2. Kaplan-Meier plot of survival rate. Significant progression free survival $(A, C)$ and overall survival $(B, D)$ rate for patients with $C D 4$ and CD8 positive endometrial carcinomas are not demonstrated.

CD8+ and CD4+ T-lymphocytes. The relationships between the rate of CD8+, CD4+ lymphocytes and clinicopathological variables were assessed and are summarized in Table 2 . Tumor grade $(P<0.001)$, myometrial invasion $(P<0.001)$, and lymph node metastasis $(P=0.002)$ were significantly associated with the rate of CD8+ lymphocytes. High numbers of CD8+ T-lymphocytes were more frequently observed in early stage than in advanced stage disease, but were not significant $(71.4 \%$ vs. $41.6 \% ; P=0.052)$. Tumor grade $(P<0.041)$ and myometrial invasion $(P=0.001)$ were significantly associated with the rate of CD4+ lymphocytes.

\section{Survival analysis}

The correlation between the positivities of CD4+, CD8+ cells and survival rate is shown in Fig. 2. In the Kaplan-Meier plot for CD4 lymphocytes, progression free survival and overall survival were not statistically significant $(P=0.388, P=0.678$, respectively) (Fig. 2A, B). Kaplan-Meier plot for CD8 revealed a slight tendency towards a higher progression free and overall survival rates for endometrial carcinoma patients with CD8 cells, although these were not statistically significant ( $P=0.080, P=0.953$, respectively) (Fig. 2C, D). 


\section{Obstetrics \& Gynecology Science}

In Kook Jung, et al. T lymphocytes as prognostic factors

\section{Discussion}

Lymphocyte infiltration in tumor tissues is considered as a host immune response to a tumor. The prognostic implication of lymphocytic infiltration has been shown in many human tumors including malignant melanoma, colorectal, esophageal, cervical carcinoma, breast, and epithelial ovarian cancer [4,5,8-11]. This survival advantage arises from sequential inflammatory and immune reactions: First, well differentiated cancer induces an inflammatory response similar to an acute injury; this is followed by sequential infiltration with dendritic cells and T-lymphocytes [12].

We revealed a prognostic significance for the tumor infiltrating T-lymphocytes in endometrial carcinoma patients. The present study showed that the proportion of CD8+ lymphocyte infiltration was negatively correlated with histologic grade, myometrial invasion and lymph node metastasis. In addition, the proportion of the CD4+ lymphocyte infiltration was negatively correlated with histologic grade and myometrial invasion.

The tumor cells secret the pro-inflammatory cytokines; consequently leukocytes of the innate and adaptive immune system, including macrophages, neutrophils, NK cells, dendritic cells, mast cells, T and B lymphocytes, infiltrate into the tumor microenvironment. In endometrial carcinoma, higher numbers of macrophages [13], neutrophils [14], B and T-lymphocytes [15], and dendritic cells [16] were infiltrated when compared with normal endometrial tissue. Among these, TILs are one of the major immune components in human solid tumors. The CD8+ suppressor/cytotoxic phenotype is mainly expressed in endometrial carcinomas, and B-lymphocyte and macrophage markers are expressed as minor subsets [17]. Each T-lymphocyte subset has a unique role in the antitumor immune response. An essential role in initiating and maintaining anticancer immune responses is played by CD4 in controlling immune responses to self and non-self antigen regulatory $\mathrm{T}$ cells [18]. In addition, cytotoxic T cells have an important role in antitumor immunity.

The infiltration of cytotoxic T-lymphocytes (CTLS) was associated with an improved survival in endometrial and ovarian cancer [3]. A high infiltration of CD8+ T cells was significantly linked with favorable prognostic factors in various tumors including colon, lung, renal cell, gastric, and ovarian cancers [19]. These studies imply that assessment of nest CD8 counts can be used clinically to measure the host-immune status in cancer patients. In endometrial cancer, the percentage of en- dothelial CD8+ T cells was associated with prognostic factors, especially invasion of up to half the myometrium [20]. On the contrary, Jackaman et al. [21] demonstrated that a low infiltration of CD8+ $T$ cells has been associated with poor prognosis, and concluded that CD8+ T cells seem to be required for reducing tumor-associated vascularity.

We show that a high rate of stromal CD8+ T-lymphocytes has a positive prognostic influence in endometrial cancer patients. Current evidence indicates that the interplay between cancer cells and stromal components is one of the most important events in tumor biology. This result has been shown in two previous studies $[3,6]$. Ino et al. [6] reported a significant difference in the progression free survival between high and low stromal CD8 groups and stromal CD8+ counts were significant prognostic factors for overall survival $(P=0.041)$. Kondratiev et al. [3] showed that the number of CD8+ lymphocytes was not significantly associated with tumor grade and stage; however, the number of CD8+ lymphocytes in the underlying stroma was significantly correlated with the occurrence of vascular invasion. CD8+ T cell infiltration in the tumor epithelium was a favorable prognostic factor in endometrial carcinoma.

A recent study that analyzed the relationship between immunohistochemical staining for CD4+ and CD8+ lymphocytes and disease progression indicated that reduced staining for CD4+ and CD8+ lymphocytes in progressive disease when compared to non-progressive disease [2].

The traditional clinicopathological prognostic variables for endometrioid-type endometrial carcinoma include International Federation of Gynecology and Obstetrics (FIGO), FIGO stage, tumor grade, myometrial invasion, lymph-vascular space status and lymph node status. Based on these prognostic factors, therapeutic stratification is warranted. Patients with unfavorable prognostic factors are currently treated with more radical surgery plus systemic lymphadenectomy and/or adjuvant radiotherapy. Further optimization and therapeutic strategy is required for patients with unfavorable prognostic factors for the improvement of clinical outcomes. In addition to the traditional prognostic variables, immunohistochemical staining for tumor infiltrating immune cells could be used for the prediction of prognosis. Therefore, further immunohistochemical evaluation of tumor infiltrating immune cells should be conducted for the development of novel therapeutic strategies for endometrial carcinoma [22].

The underlying mechanisms of activation and distribution of 


\title{
Obstetrics \& Gynecology Science
}

\author{
Vol. 57, No. 4, 2014
}

activated TILs in endometrial carcinoma still remains unclear. Several possible mechanisms have been reported. After presentation of a tumor-specific antigen by antigen-presenting cells or by tumor cells themselves, lymphocyte activation and proliferation may occur [23]. Another mechanism suggested by Kummer et al. [24] is that cytotoxic T-lymphocytes may be activated by a general inflammatory reaction, such as the release of activating cytokines. Ohno et al. [19] showed that COX-2 may be a limiting factor that controls the degree of CD8+ $T$ cell infiltration in tumor tissues. Indoleamine-2,3dioxygenase (IDO) inhibits T-cell functions in cancer, and Ino et al. [6] showed that high IDO expression was correlated with reduced infiltration of T-lymphocytes in endometrial cancer and that a low percentage of $T$ cells was associated with poor clinical outcome. Low grade and early stage endometrial cancer patients often showed a high proportion of CD8+ cytotoxic T-lymphocytes. The difference in CD8+ infiltration according to histologic grade might result from the factors produced by the cancer cells themselves or from major histocompatibility complex expression. Further description of the differences in infiltration of T cells in low grade vs. high grade tumors is needed [25]. Previously, van der Horst et al. [2] showed that both the presence of TILs and positive progesterone receptor were correlated with better prognosis. These results imply that progesterone might have a potential role in attracting TILs in endometrial cancer [2].

Our study has some limitations. First, demographic data include the limited number of patients, and unintended skewed patient distributions, especially in terms of stage and lymph node metastasis. Next, tissue microassay is powerful technology emerged recently. Each tissue sample on a tissue microarray slide is treated in an identical manner. This uniformity improves the specificity and sensitivity of staining, reproducibility of the staining reaction and reliability of the interpretation [26]. Instead, we used immunohistochemical stainings for the assessment of CD4 and CD8 distribution.

In conclusion, our results showed that the proportion of stromal infiltration of T-lymphocytes was correlated with clinicopathologic factors such as histologic grade, myometrial invasion and lymph node metastasis in endometrial carcinoma. Although the mechanism of tumor infiltrating T-lymphocyte activation and distribution of activated lymphocytes in endometrial carcinoma remains to be elucidated, our findings suggest that tumor-infiltrating T-lymphocytes may be used as pathologic prognostic factors in endometrial carcinoma.

\section{Conflict of interest}

No potential conflict of interest relevant to this article was reported.

\section{Acknowledgments}

This study was supported by a grant of the Health Fellowship Foundation.

\section{References}

1. Jung KW, Won YJ, Kong HJ, Oh CM, Seo HG, Lee JS. Cancer statistics in Korea: incidence, mortality, survival and prevalence in 2010. Cancer Res Treat 2013;45:1-14.

2. Van der Horst PH, Wang Y, Vandenput I, Kuhne LC, Ewing PC, van ljcken WF, et al. Progesterone inhibits epithelialto-mesenchymal transition in endometrial cancer. PLoS One 2012;7:e30840.

3. Kondratiev S, Sabo E, Yakirevich E, Lavie O, Resnick MB. Intratumoral CD8+ $T$ lymphocytes as a prognostic factor of survival in endometrial carcinoma. Clin Cancer Res 2004;10:4450-6.

4. Zhang L, Conejo-Garcia JR, Katsaros D, Gimotty PA, Massobrio M, Regnani G, et al. Intratumoral T cells, recurrence, and survival in epithelial ovarian cancer. N Engl J Med 2003;348:203-13.

5. Naito Y, Saito K, Shiiba K, Ohuchi A, Saigenji K, Nagura H, et al. CD8+ T cells infiltrated within cancer cell nests as a prognostic factor in human colorectal cancer. Cancer Res 1998;58:3491-4.

6. Ino K, Yamamoto E, Shibata K, Kajiyama H, Yoshida N, Terauchi $M$, et al. Inverse correlation between tumoral indoleamine 2,3-dioxygenase expression and tumor-infiltrating lymphocytes in endometrial cancer: its association with disease progression and survival. Clin Cancer Res 2008; 14:2310-7.

7. Kobayashi N, Hiraoka N, Yamagami W, Ojima H, Kanai $Y$, Kosuge T, et al. FOXP3+ regulatory T cells affect the development and progression of hepatocarcinogenesis. Clin Cancer Res 2007;13:902-11.

8. Bontkes HJ, de Gruijl TD, Walboomers JM, van den Muysenberg AJ, Gunther AW, Scheper RJ, et al. Assessment 


\section{Obstetrics \& Gynecology Science}

In Kook Jung, et al. T lymphocytes as prognostic factors

of cytotoxic T-lymphocyte phenotype using the specific markers granzyme B and TIA-1 in cervical neoplastic lesions. Br J Cancer 1997;76:1353-60.

9. Yakirevich E, Izhak OB, Rennert G, Kovacs ZG, Resnick $M B$. Cytotoxic phenotype of tumor infiltrating lymphocytes in medullary carcinoma of the breast. Mod Pathol 1999;12:1050-6.

10. Schumacher K, Haensch W, Roefzaad C, Schlag PM. Prognostic significance of activated CD8(+) T cell infiltrations within esophageal carcinomas. Cancer Res 2001;61:3932-6.

11. Clark WH Jr, Elder DE, Guerry D 4th, Braitman LE, Trock BJ, Schultz D, et al. Model predicting survival in stage I melanoma based on tumor progression. J Natl Cancer Inst 1989;81:1893-904.

12. Clark CE, Hingorani SR, Mick R, Combs C, Tuveson DA, Vonderheide $\mathrm{RH}$. Dynamics of the immune reaction to pancreatic cancer from inception to invasion. Cancer Res 2007;67:9518-27.

13. Ohno S, Ohno Y, Suzuki N, Kamei T, Koike K, Inagawa $\mathrm{H}$, et al. Correlation of histological localization of tumor-associated macrophages with clinicopathological features in endometrial cancer. Anticancer Res 2004;24(5C):3335-42.

14. Wallace $A E$, Sales KJ, Catalano RD, Anderson RA, Williams AR, Wilson MR, et al. Prostaglandin F2alphaF-prostanoid receptor signaling promotes neutrophil chemotaxis via chemokine (C-X-C motif) ligand 1 in endometrial adenocarcinoma. Cancer Res 2009;69:572633.

15. Giatromanolaki A, Bates GJ, Koukourakis MI, Sivridis $E$, Gatter KC, Harris AL, et al. The presence of tumorinfiltrating FOXP3+ lymphocytes correlates with intratumoral angiogenesis in endometrial cancer. Gynecol Oncol 2008;110:216-21.

16. Wallace AE, Gibson DA, Saunders PT, Jabbour HN. Inflammatory events in endometrial adenocarcinoma. J Endocrinol 2010;206:141-57.

17. Ferguson A, Moore M, Fox H. Expression of MHC products and leucocyte differentiation antigens in gynaeco- logical neoplasms: an immunohistological analysis of the tumour cells and infiltrating leucocytes. $\mathrm{Br} J$ Cancer 1985;52:551-63.

18. Yamaguchi T, Sakaguchi S. Regulatory T cells in immune surveillance and treatment of cancer. Semin Cancer Biol 2006;16:115-23.

19. Ohno Y, Ohno S, Suzuki N, Kamei T, Inagawa H, Soma G, et al. Role of cyclooxygenase-2 in immunomodulation and prognosis of endometrial carcinoma. Int J Cancer 2005; 114:696-701.

20. Ribatti D, Nico B, Finato N, Crivellato E. Tryptase-positive mast cells and CD8-positive T cells in human endometrial cancer. Pathol Int 2011;61:442-4.

21. Jackaman C, Bundell CS, Kinnear BF, Smith AM, Filion $P$, van Hagen $D$, et al. IL-2 intratumoral immunotherapy enhances CD8+ T cells that mediate destruction of tumor cells and tumor-associated vasculature: a novel mechanism for IL-2. J Immunol 2003;171:5051-63.

22. De Jong RA, Leffers $N$, Boezen HM, ten Hoor KA, van der Zee $A G$, Hollema $H$, et al. Presence of tumorinfiltrating lymphocytes is an independent prognostic factor in type I and II endometrial cancer. Gynecol Oncol 2009; 114:105-10.

23. Germain RN, Margulies DH. The biochemistry and cell biology of antigen processing and presentation. Annu Rev Immunol 1993;11:403-50.

24. Kummer JA, Kamp AM, Tadema TM, Vos W, Meijer CJ, Hack CE. Localization and identification of granzymes $A$ and B-expressing cells in normal human lymphoid tissue and peripheral blood. Clin Exp Immunol 1995;100:16472.

25. Han $L Y$, Fletcher MS, Urbauer DL, Mueller $P$, Landen $C N$, Kamat AA, et al. HLA class I antigen processing machinery component expression and intratumoral T-Cell infiltrate as independent prognostic markers in ovarian carcinoma. Clin Cancer Res 2008;14:3372-9.

26. Arafa M, Boniver J, Delvenne P. Progression model tissue microarray (TMA) for the study of uterine carcinomas. Dis Markers 2010;28:267-72. 